\title{
Gold-Hyperdoped Germanium with Room-Temperature Sub-Band-Gap Optoelectronic Response
}

\author{
Hemi H. Gandhi, ${ }^{1, * * *,+\dagger}$ David Pastor, ${ }^{1,2, \dagger^{+}, * *, \dagger \dagger}$ Tuan T. Tran, ${ }^{3,4}$ S. Kalchmair $\odot,{ }^{1}$ L.A. Smilie, ${ }^{3}$ \\ Jonathan P. Mailoa, ${ }^{5}$ Ruggero Milazzo $\odot,{ }^{6}$ Enrico Napolitani $\odot,{ }^{6}$ Marco Loncar, ${ }^{1}$ James S. Williams, ${ }^{3}$ \\ Michael J. Aziz@ $,^{1, \ddagger}, * *$ and Eric Mazur ${ }^{1, \S, * *}$ \\ ${ }^{1}$ Harvard John A. Paulson School of Engineering and Applied Sciences, Cambridge, Massachusetts 02138, USA \\ ${ }^{2}$ Departamento de Estructura de la Materia, Física térmica y Electrónica, Facultad de Ciencias Físicas, \\ Universidad Complutense de Madrid, Madrid 28040, Spain \\ ${ }^{3}$ Department of Electronic Materials Engineering, Research School of Physics and Engineering, Australian \\ National University, Canberra, Australian Capital Territory 0200, Australia \\ ${ }^{4}$ Department of Physics and Astronomy, Angström Laboratory, Uppsala University, Box 516, SE-751 20 Uppsala, \\ Sweden \\ ${ }^{5}$ Robert Bosch LLC, Cambridge, Massachusetts 02138, USA \\ ${ }^{6}$ Dipartimento di Fisica e Astronomia, Università di Padova and CNR-IMM, Via Marzolo 8, I-35131 Padova, Italy
}

(Received 19 March 2020; revised 27 August 2020; accepted 9 October 2020; published 16 December 2020)

Short-wavelength-infrared (SWIR; 1.4-3.0 $\mu \mathrm{m}$ ) photodetection is important for various applications. Inducing a low-cost silicon-compatible material, such as germanium, to detect SWIR light would be advantageous for SWIR applications compared with using conventional (III-V or II-VI) SWIR materials. Here, we present a scalable nonequilibrium method for hyperdoping germanium with gold for dopantmediated SWIR photodetection. Using ion implantation followed by nanosecond pulsed laser melting, we obtain a single-crystal material with a peak gold concentration of $3 \times 10^{19} \mathrm{~cm}^{-3}\left(10^{3}\right.$ times the solubility limit). This hyperdoped germanium has fundamentally different optoelectronic properties from those of intrinsic and conventionally doped germanium. This material exhibits sub-band-gap absorption of light up to wavelengths of at least $3 \mu \mathrm{m}$, with a sub-band-gap optical absorption coefficient comparable to that of commercial SWIR photodetection materials. We show that germanium hyperdoped with gold exhibits sub-band-gap SWIR photodetection at room temperature, in contrast with previous doped-germanium photodetector studies, which only show a low-temperature response. This material is a potential pathway to low-cost room-temperature silicon-compatible SWIR photodetection.

DOI: 10.1103/PhysRevApplied.14.064051

\section{INTRODUCTION}

Many emerging technologies, including industrial and medical imaging, astronomy, biological and chemical sensing, LIDAR, and surveillance, rely on shortwavelength-infrared (SWIR; 1.4-3.0 $\mu \mathrm{m}$ ) photodetection [1-5]. The elemental semiconductors silicon ( $\mathrm{Si}$ ) and germanium (Ge) do not absorb over the majority of the SWIR spectral range, which consists of photons that have energies below the band gap of these materials. Currently, state-of-the-art commercial SWIR photodetectors

\footnotetext{
*hemi.gandhi@alumni.harvard.edu

†dpastor@seas.harvard.edu and dpastor@fis.ucm.es

†maziz@harvard.edu

§mazur@seas.harvard.edu

** To whom all correspondence (inquiry) should be addressed.

${ }^{\dagger \dagger}$ H.H.G. and D.P. are co-first authors and contributed equally to this work.
}

use narrow-band-gap III-V or II-VI semiconductors [1] [e.g., $\mathrm{In}_{1-x} \mathrm{Ga}_{x} \mathrm{As}, \mathrm{InAs}, \mathrm{Pb}_{1-x} \mathrm{Se}_{x}, \mathrm{Hg}_{1-x} \mathrm{Cd}_{x} \mathrm{Te}$ ] that are heterogeneously integrated with Si CMOS electronics. Three main issues limit the applicability of such photodetectors $[1,6,7]$. First, III-V and II-VI semiconductors are chemically incompatible with Si CMOS processing, limiting the size of final photodetector focal plane arrays, and are often expensive or toxic. Second, their heterogeneous integration is a complex low-throughput process that further increases the final device cost. Third, photodetectors made from the majority of these materials must be cooled to low temperature to attain satisfactory signal-tonoise ratios. Recent reports present alternative approaches to using III-V or II-VI materials for SWIR photodetection. These reports utilize recently developed materials, ranging from two-dimensional materials [8-12] to metasurfaces $[13,14]$ to nanocrystals $[10,15]$ to organic $[16]$ and bioinspired [17] materials. It is not clear, however, how these materials might be integrated with existing Si-based devices. The lack of a low-cost nontoxic Si-compatible 
material capable of room-temperature SWIR photodetection constrains applications of SWIR photodetection.

Conventional low-cost and nontoxic semiconductors, such as $\mathrm{Si}$ or Ge-on-Si, do not absorb the majority of the SWIR spectrum, which consists of photons that have energies below the band gap of these materials. However, absorption in $\mathrm{Si}$ or Ge-on-Si can be extended to longer wavelengths by redshifting the conduction band to valence band transitions through strain or alloying, or by creating sub-band-gap extrinsic absorption transitions between sub-band-gap states and the conduction and valence bands through the incorporation of lattice defects or dopants [18-21].

Here, we report dopant-mediated sub-band-gap photodetection in Ge doped with supersaturated concentrations of gold $(\mathrm{Au})$. We use a scalable nonequilibrium hyperdoping method [22], consisting of ion implantation of $\mathrm{Au}$ in $\mathrm{Ge}$ followed by nanosecond pulsed laser melting (PLM) induced rapid solidification. This process results in a single-crystal material with a peak Au concentration of $3 \times 10^{19} \mathrm{~cm}^{-3}$, a factor of $10^{3}$ greater than the $\mathrm{Ge}: \mathrm{Au}$ solubility limit [23]. Supersaturation increases the dopantmediated sub-band-gap absorption coefficient, $\alpha$, to values comparable to those of commercial SWIR III-IV and II-VI materials [4,24]. Using hyperdoped Ge:Au, we demonstrate a proof-of-concept prototype photodetector with a room-temperature sub-band-gap SWIR optoelectronic response.

While recent sub-band-gap photodetection studies have focused on $\mathrm{Si}$, we work with Ge because of its higher carrier mobility [18,25-28]. Previous studies of dopantmediated Ge photodetectors incorporating various dopants ( $\mathrm{S}, \mathrm{Te}, \mathrm{Zn}, \mathrm{B}, \mathrm{Cu}, \mathrm{Cd}, \mathrm{Zn}, \mathrm{Au}$ ) through various doping methods have reported sub-band-gap responses only at low temperature, which is impractical for many SWIR applications [27-31]. We select $\mathrm{Au}$ as a dopant because $\mathrm{Ge}: \mathrm{Au}$ has several properties that make it promising for a dopantmediated sub-band-gap photodetector. Au is a deep-level dopant and, relative to Ge with shallow-level dopants $[32,33]$, Ge:Au demonstrates a lower thermal ionization at room temperature, reducing background free-carrier concentrations and improving device signal-to-noise ratios [27,28]. Among deep-level Ge dopants, Au uniquely has self-compensating defect levels [34] (see Fig. S1 within the Supplemental Material [35]) and a relatively high equilibrium solubility limit [23]. Self-compensating defect levels reduce background free-carrier concentrations, and a higher equilibrium solubility limit permits attaining even higher concentrations through hyperdoping [36,37], leading to a higher sub-band-gap absorption coefficient.

Previous efforts to develop sub-band-gap photodetection in Ge:Au have been limited by low Au-doping concentrations. These studies, primarily carried out in the 1950s-1970s, used conventional near-equilibrium doping methods that did not produce doping concentrations above the Ge:Au solubility limit $\left(2.8 \times 10^{16} \mathrm{~cm}^{-3}\right)[23,27]$. Consequently, these Ge:Au photodetectors have a very low sub-band-gap absorption coefficient $\left(\alpha \approx 0.1 \mathrm{~cm}^{-1}\right)$ [27, $28,35]$, and at room-temperature the background freecarrier concentration overwhelms the photosignal [30]. Such detectors, therefore, only operate under cooled conditions, typically between 4 and $78 \mathrm{~K}$. Here, we show that hyperdoped Ge:Au has fundamentally different properties from those of conventional $\mathrm{Ge}: \mathrm{Au}$, namely, a much larger sub-band-gap absorption coefficient comparable to that of direct-band-gap semiconductors and room temperature sub-band-gap SWIR photodetection [38]. We note that significant progress has been made on Ge-Sn alloys [39, 40], which have recently demonstrated room-temperature SWIR photodetection [41-44] and lasing [21,45], but which operate by a fundamentally different sub-bandgap absorption mechanism than that of the Au-dopant mediation investigated here. Ge-Sn alloying ( $\sim 9 \% \mathrm{Sn})$ creates high compressive strain, which can induce a redshifted direct-band-gap transition $[39,40]$. Au hyperdoping (approximately $0.07 \% \mathrm{Au}$ in our high-dose sample) introduces a nonequilibrium concentration of sub-band-gap defect states, which facilitate sub-band-gap absorption.

The Ge hyperdoping method used here could be transferred to an existing Si-based optoelectronic device fabrication process. Ge-on-Si photodetectors have already successfully extended the detection edge of Si-based devices to telecom wavelengths [46]. Adding two additional steps, ion implantation and PLM, to an existing Ge-on-Si photodetector fabrication process could further extend detection to cover the entire SWIR spectrum. As these two fabrication steps use standard semiconductor industry tools, ion implanters and pulsed lasers, the development of hyperdoped Ge for SWIR-photodetection applications would be relatively straightforward. Although we fabricate the prototype hyperdoped Ge photodetector presented here on a Ge wafer, the method can be transferred to strained Geon-Si substrates with no loss of crystallinity [47]. Because nanosecond PLM only significantly heats the top surface of a wafer (of order $1 \mu \mathrm{m}$ ), it can be restricted to userdefined areas and applied as the last processing step of an existing optoelectronic device fabrication process to limit unintended Au diffusion [26].

\section{METHODS}

\section{A. Hyperdoping experimental design}

We implant two-side polished (100) Czochralski-grown 5-10 $\Omega \mathrm{cm}$ resistivity $n$-type $\mathrm{Ge}: \mathrm{Sb}$ wafers with $110 \mathrm{keV}$ ${ }^{197} \mathrm{Au}^{-}$ions to a dose of $10^{14}$ or $6 \times 10^{14} \mathrm{~cm}^{-2}$. The projected range for both doses is $27 \mathrm{~nm}$. We note that the low dose is at least 6 times the minimumimplant dose that is shown to produce full amorphization of $\mathrm{Ge}$ from implant species implanted at a comparable energy range $[48,49]$. We perform all 
implantations at liquid-nitrogen temperature to suppress ion-beam-induced porosity and ion-induced annealing [50,51]. We orient all substrates $7^{\circ}$ with respect to the [100] crystal axis to minimize ion channeling.

To characterize the resulting amorphous layer, we perform channeling Rutherford backscattering spectrometry (RBS) on the samples with a $2 \mathrm{MeV} \mathrm{He}^{+}$ion beam aligned along the [100] axis. We evaluate ion-scattering measurements by simulating RBS spectra using the SIMNRA simulation program [43]. The program, in general, considers only single scattering events, allowing analytical formulae to be used for the calculation of the scattering parameters, hence vastly speeding up the simulation. Also, we choose SRIM for the electronic stopping power data because SRIM is a semiempirical database that relies on available experimental stopping powers. For $\mathrm{MeV}$ ion scattering, the electronic energy loss provided by SRIM is very reproducible [52]. The SIMNRA simulations indicate that the $10^{14}$ and $6 \times 10^{14} \mathrm{~cm}^{-2}$ implanted samples have an amorphous layer thickness of 47 and $66 \mathrm{~nm}$, respectively.

These SIMNRA simulations assume a two-layer system (layer 1, Ge:Au; layer 2, amorphous pure $\mathrm{Ge}$ ) and apply a diffuse interface, as well as finite detector resolution, both of which give rise to broadening of layer edges. The simulations, therefore, effectively model a gradual transition from the amorphous to crystalline layers that occurs in asimplanted samples. This gives rise to the tail at around channel 370 in Fig. 1(b) in the simulated as-implanted case (dashed red curve).

To restore crystallinity after implantation damage, we then laser melt each implanted sample with a $355 \mathrm{~nm}$ $0.49 \mathrm{~J} / \mathrm{cm}^{2}$ single $4 \mathrm{~ns}$ FWHM pulse from a Nd:YAG laser. We choose this fluence to reach a maximum melt depth beyond the implant-amorphized region of each sample [the melt depth is predicted to be $210 \mathrm{~nm}$ using numerical solutions to the heat equation; Fig. S2(d) within the Supplemental Material [35]]. In this scenario, the melt front reaches the underlying crystalline substrate and leads to single-crystal epilayer regrowth. Throughout the process, we measure sample melt duration through time-resolved reflectivity (TRR) [53], using the setup depicted in Fig. S2(a) within the Supplemental Material [35]. TRR is enabled by a low-power $\mathrm{Ar}^{+}$ion probe laser and a photodiode, as shown in the setup. During melting, the probe laser reflects off the sample and onto the photodiode. Molten $\mathrm{Ge}$, which is metallic, has a considerably higher reflectivity (a)

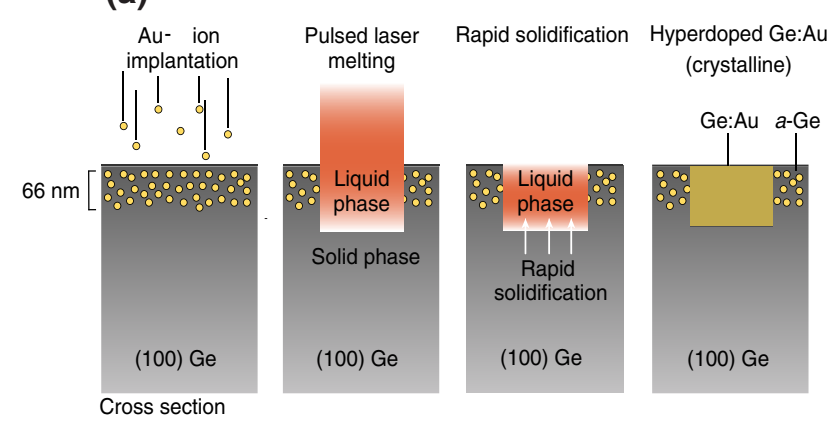

(c)

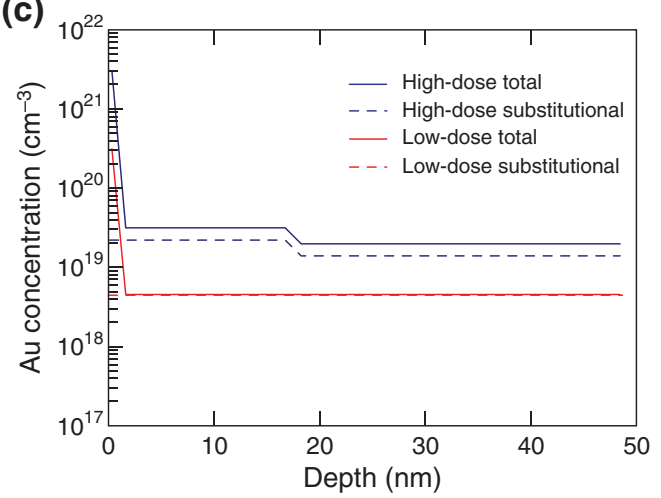

(b)

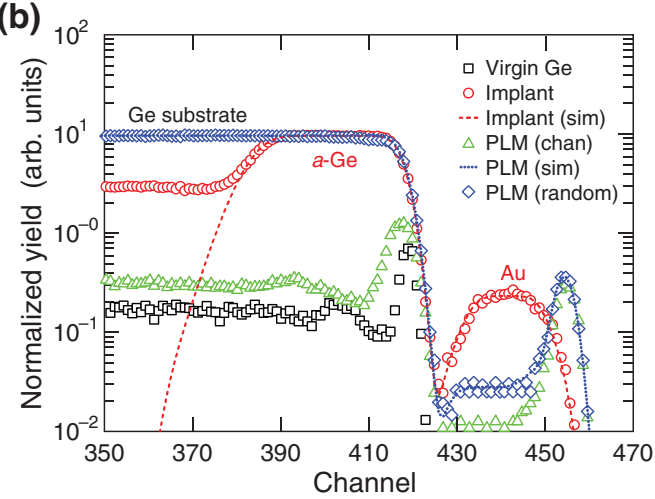

(d)

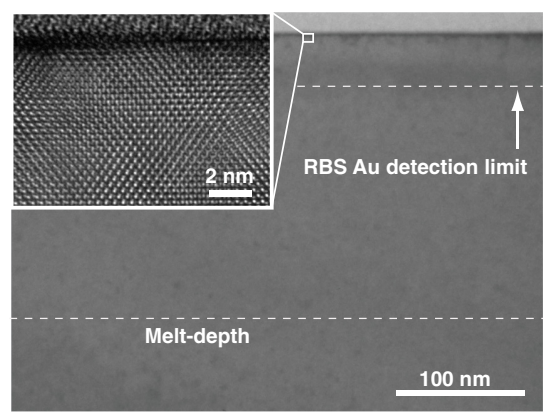

FIG. 1. Structural properties of hyperdoped Ge. (a) Schematic of the hyperdoping process: (i) ion implantation introduces the desired dopant quantity into the host material, (ii) pulsed laser melts implantation-induced lattice damage, and (iii) epitaxial rapid solidification traps a supersaturated dopant concentration into single-crystalline phase. (b) RBS spectra of virgin Ge and implanted high-dose $\left(6 \times 10^{14} \mathrm{~cm}^{-2}\right)$ Ge before and after PLM. All spectra shown are channeled measurements, except the random PLM spectra indicated and its simulation. (c) Inferred concentration-depth profiles of low- $\left(10^{14} \mathrm{~cm}^{-2}\right)$ and high-dose $\left(6 \times 10^{14} \mathrm{~cm}^{-2}\right.$ dose $)$ hyperdoped Ge samples. (d) XTEM and high-resolution (HR) XTEM (inset) micrographs of the high-dose hyperdoped Ge sample. 
than those of amorphous or crystalline Ge, both of which are semiconductors $[48,49]$. Hence, during melting, the amount of probe light reflected by the sample and collected by the photodiode (in a melt trace) increases significantly. A typical TRR melt trace is shown in Fig. S2(b) within the Supplemental Material [35].

During PLM of the implanted samples, time-resolved reflectivity measurements indicate the following melt durations: between 33 and $54 \mathrm{~ns}$ for the $10^{14} \mathrm{~cm}^{-2}$ $0.49 \mathrm{~J} / \mathrm{cm}^{2}$ samples and 39-66 ns for the $6 \times 10^{14} \mathrm{~cm}^{-2}$ $0.49 \mathrm{~J} / \mathrm{cm}^{2}$ samples. These measured melt durations are consistent with our numerical simulations. A typical reflectivity trace is shown in Fig. S2(c) within the Supplemental Material [35].

\section{B. Structural measurements}

To examine the impact of PLM on subsurface implantation damage, we carry out bright-field and high-resolution cross-section transmission electron microscopy (XTEM) at $200 \mathrm{keV}$. We prepare the samples for XTEM using a focused ion beam and in situ lift out.

To characterize sample crystallinity and the dopant profile, we perform channeled and random-mode RBS. The random-mode measurements are performed in the same geometry as that of the channeled measurements, but with the sample rotated about $7^{\circ}$ from its surface normal. We determine the as-implanted dose and thickness of each sample by simulating and fitting the as-implanted spectra with the SIMNRA program. For the PLM spectra, we determine the total $\mathrm{Au}$ concentration profile by simulating the random spectra using SIMNRA. To determine the substitutional $\mathrm{Au}$ concentration profile, we multiply the total $\mathrm{Au}$ concentration profile with the substitutional factor $S$, which can be calculated using the following equation [54]:

$$
S=\left(1-\frac{Y_{\mathrm{Au} / \text { channeled }}}{Y_{\mathrm{Au} / \text { random }}}\right) /\left(1-\frac{Y_{\mathrm{Ge} / \text { channeled }}}{Y_{\mathrm{Ge} / \text { random }}}\right),
$$

where $Y_{X / \text { channeled }}$ and $Y_{X / \text { random }}$ are the scattering yields from the $X$ elements at channeled and random geometries, respectively. In our study, the scattering yields of the channeled and random spectra are taken at the sample depth, excluding the surface peak.

\section{Optical measurements}

We characterize sample absorptance by performing reflectance and transmittance measurements using a UVnear-infrared (NIR) spectrophotometer and FTIR. To minimize gas-absorption lines in absorptance data, we purge the FTIR chamber with liquid $\mathrm{N}_{2}$ before all measurements. We model the hyperdoped Ge:Au layer using the twolayer thin-film-stack methodology described previously [26]. In the model, we assume the hyperdoped Ge:Au layer is $50 \mathrm{~nm}$ thick, use a measured absorptance value of 0.027 at $2.4 \mu \mathrm{m}$, and assume an air-virgin Ge interface normal-incidence reflection coefficient of 0.3366 .

\section{Photodetector fabrication}

We fabricate Ge:Au photodetectors as follows. To create a bottom contact to the $n$-type wafer substrate, we coimplant $\mathrm{Sb}$ and $\mathrm{P}$ on one side of the wafer, rapidly thermal anneal the sample, and perform metal evaporation, as described elsewhere [55]. On the wafer side opposite the $n^{+}$layer, we form the $p^{+}$Ge:Au layer via the hyperdoping process detailed above. Using photolithography and $\mathrm{SF}_{6}$ reactive ion etching, we then form a mesa structure out of the $p^{+}$layer to electrically isolate the region from the rest of the substrate. The mesas are $1 \mu \mathrm{m}$ deep and $1 \times 1 \mathrm{~mm}^{2}$ in area. We then use photolithography, electronbeam metal evaporation, and lift off to form 200 -nm-thick Al-bar contacts on opposite sides of the mesa structure to create the top photodetector device contacts. All contacts are ohmic, as shown in Fig. S6 within the Supplemental Material [35]. We attach the bottom contact to a printed circuit board (PCB) with silver paste and wire bond the top contacts to the PCB. We fabricate Ge:Sb control photodetectors in a similar procedure to that of the $\mathrm{Ge}: \mathrm{Au}$ photodetectors, with the hyperoped-Au layer replaced with Ge:Sb implanted with an energy of $65 \mathrm{keV}$.

\section{Optoelectronic response measurements}

We take precautions to avoid a false sub-band-gap photosignal in the laser measurement setup details described above. To this end, we place a $300-\mu \mathrm{m}$-thick Ge wafer in the laser path to filter out above-band-gap light. To prevent ambient light from inducing a false photoconductive signal, we perform measurements in complete darkness.

To determine the spectral photoresponse of the photodetectors, we substitute each photodetector for the detector in a FTIR spectrometer. In this FTIR photoconductivity setup, we connect a photodetector to a transimpedance amplifier (TIA) for amplification of the photocurrent. No bias voltage is applied to the photodetector during measurements. The FTIR resolution is set to $100 \mathrm{~cm}^{-1}$. To reduce noise, we set the band-pass filter of the TIA to the range from 0.1 to $10 \mathrm{kHz}$. We obtain the photoconductive spectrum in arb. units, since the incident power from the Globar light source is unknown. The measured photoresponse of each detector is normalized to the emission spectrum of the Globar light source. When measuring dark noise, we block illumination from the FTIR spectrometer just before the objective lens to ensure that no light reaches the photodetector.

\section{RESULTS}

\section{A. Germanium hyperdoping process}

To fabricate hyperdoped Ge [Fig. 1(a)], we first implant $n$-type (100) $\mathrm{Ge}$ wafers with $110 \mathrm{keV}{ }^{197} \mathrm{Au}^{-}$ions to 
a dose of $10^{14}$ or $6 \times 10^{14} \mathrm{~cm}^{-2}$. In the implanted $10^{14} \mathrm{~cm}^{-2}$ samples, implanted $\mathrm{Au}$ is distributed throughout the entire the 47-nm-thick amorphous layer. In the implanted $6 \times 10^{14} \mathrm{~cm}^{-2}$ dose samples, Au is distributed within the first $50 \mathrm{~nm}$ of a 66-nm-thick amorphous layer. To restore crystallinity after implantation-induced amorphization, we laser melt each implanted sample with a $355 \mathrm{~nm} 0.49-\mathrm{J} / \mathrm{cm}^{2}$ single $4 \mathrm{~ns}$ FWHM pulse from a $\mathrm{Nd}$ :YAG laser. We choose this fluence to reach a melt depth of $210 \mathrm{~nm}$ (predicted by numerical solutions of the heat equation, as shown in Fig. S2(d) within the Supplemental Material [35], which is expected to be safely beyond the deep tail of the implant distribution and beyond the implant-amorphized and implant-damaged regions of each sample [56]. In this scenario, the melt front reaches the underlying perfect crystalline substrate, permitting defect-free single-crystal epitaxial regrowth. The laser irradiation conditions are chosen so that the subsequent solidification speed is in the order of $1 \mathrm{~m} / \mathrm{s}$, which is slow enough to permit defect-free crystal growth, but fast enough to cause solute trapping, or nonequilibrium impurity incorporation during rapid solidification [57], resulting in a hyperdoped supersaturated single-crystal material.

\section{B. Structural properties}

To characterize Au distribution in the hyperdoped samples, we carry out channeling and random-mode RBS measurements on virgin, as-implanted, and hyperdoped (after PLM) Ge wafers. To quantify the structure and composition of the samples, we fit the results of simulations, using the SIMNRA simulation program, to the experimental spectra. The experimental RBS spectra of the $6 \times 10^{14} \mathrm{~cm}^{-2}$ Au samples are shown in Fig. 1(b). All RBS spectra are of channeling-mode measurements, except the random PLM spectrum (specifically indicated in the legend) and the SIMNRA simulation [58]. The SIMNRA simulation (described in Sec. II) is performed to obtain the amorphous layer thickness and Au profiles. In RBS measurements, the channeling mode probes crystal defects and atomic location of $\mathrm{Au}$, whereas the random mode probes the total distribution of atoms, including interstitial and substitutional atoms. In the channeling-mode as-implanted spectrum (red circles), two scattering peaks are visible. The first peak, from channels 380 to 420 , is the scattering yield from Ge atoms within the implanted layer. The second peak, from channels 425 to 459 , is the scattering yield from $\mathrm{Au}$ atoms. The simulated spectrum (red dashes) indicates that $\mathrm{Au}$ is distributed within the first $50 \mathrm{~nm}$ of a 66-nm-thick amorphous layer. For the hyperdoped spectrum taken in the channeled mode (green triangles), the minimum scattering yield of the Ge substrate $\left(\chi_{\mathrm{min} / \mathrm{Ge}}\right)$ is very close to that of a virgin Ge wafer (black squares), suggesting that the hyperdoped samples after laser melting are of excellent crystalline quality. Regarding the distribution of $\mathrm{Au}$, between channels 425 and 459, the significant difference in Au scattering yield between the random and channeled spectra (blue diamonds vs green triangles) indicates that the majority of implanted $\mathrm{Au}$ atoms are located on substitutional Ge lattice sites after PLM. From the Au peak towards lower channel numbers, the channeled and random PLM scattering yields decay quickly and reach a minimum near channel 425 (just before the Ge substrate peak). This decay indicates that the majority of $\mathrm{Au}$ in the hyperdoped sample is trapped within the top $50 \mathrm{~nm}$.

Figure 1(c) shows the total and substitutional $\mathrm{Au}$ concentration-depth profiles for the low- $\left(10^{14} \mathrm{~cm}^{-2}\right)$ and high-dose $\left(6 \times 10^{14} \mathrm{~cm}^{-2}\right)$ samples. The depth profiles are obtained from simulating the RBS spectra. Since any Au signal beyond $50 \mathrm{~nm}$ in depth is overwhelmed by the large Ge substrate signal, small concentrations of $\mathrm{Au}$ are to be expected beyond $50 \mathrm{~nm}$, as explained below. The total $\mathrm{Au}$ concentrations about $10 \mathrm{~nm}$ below the Au surface peak for the low-dose and high-dose samples are $4 \times 10^{18}$ and $3 \times 10^{19} \mathrm{~cm}^{-3}$, respectively. These values are several orders of magnitude higher than the Ge:Au solid solubility limit of $2.8 \times 10^{16} \mathrm{~cm}^{-3}$ and the highest previously reported Ge:Au concentration, which is obtained by rapid quenching at $1131 \mathrm{~K}$, of $1.5 \times 10^{17} \mathrm{~cm}^{-3}$ [23]. In the observable depth range from below the surface peak to $50 \mathrm{~nm}$, we find that $100 \%$ and $70 \%$ of the incorporated $\mathrm{Au}$ atoms are substitutional in the low- and high-dose samples, respectively.

Directly after implantation, the RBS profile of the highdose sample indicates that all implanted $\mathrm{Au}$ is distributed within the first $50 \mathrm{~nm}$ of an amorphous layer $66 \mathrm{~nm}$ thick (as described in Sec. III A). Dividing the integrated total concentration depth profile after PLM by the measured implanted dose from RBS suggests that $(59 \pm 1) \%$ of the implanted $\mathrm{Au}$ dose is retained between 0 and $50 \mathrm{~nm}$. The missing fraction, $(41 \pm 1) \%$, of the implant dose (i.e., $2.6 \times 10^{14} \mathrm{Au}$ atoms $/ \mathrm{cm}^{-2}$ ) either ends up at a depth beyond $50 \mathrm{~nm}$ or evaporates from the surface during the PLM process. Notably, the maximum layer thickness to which Au can diffuse during PLM is equal to the melt depth, which is predicted to be $210 \mathrm{~nm}$ from the numerical solution of the heat equation. The hyperdoped layer thickness is therefore likely to be somewhere between 50 and $210 \mathrm{~nm}$ thick. To probe the Au distribution beyond the RBS depth-resolution limit, we carry out secondary ion mass spectrometry (SIMS) using a $7.25 \mathrm{keV}$ Cs ion beam and collect ${ }^{197} \mathrm{Au}^{-}$secondary ions (Fig. S10 within the Supplemental Material [35]). The SIMS measurements are less accurate than the RBS measurements due to SIMS depth-resolution issues, but qualitatively confirm the redistribution of $\mathrm{Au}$ after laser melting and allow an upper bound of the retained $\mathrm{Au}$ dose beyond $50 \mathrm{~nm}$ to be established. We estimate that the Au dose beyond $50 \mathrm{~nm}$ for the low-dose and high-dose laser-melted samples are 
less than $5.8 \times 10^{12}(5.8 \%$ of the implanted $\mathrm{Au})$ and $5.4 \times 10^{13} \mathrm{~cm}^{-2}(9.0 \%$ of the implanted $\mathrm{Au})$, respectively.

XTEM micrographs of the high-dose hyperdoped sample [Fig. 1(d)] show that the entire sample is single crystalline. No extended defects, secondary phases, or cellular breakdown features are visible in the micrograph. Furthermore, the entire region above the predicted melt depth, which includes the hyperdoped Ge:Au layer, appears identical to the virgin substrate beneath the melt depth. The micrographs demonstrate that PLM fully restores the crystallinity of the implantation-damaged region; this conclusion is supported by the RBS spectra in Fig. 1(b).

Two other features of the micrograph are worth noting. First, a mottling of small dark spots is visible throughout the micrograph. These spots are characteristic of the focused-ion-beam damage produced during Ge XTEM sample preparation $[59,60]$. Because these spots are found throughout the specimen and beyond the melt depth, we conclude that they do not arise from the hyperdoping process. Second, the continuous dark surface layer visible at the top of the micrograph can be attributed to a surface oxide producing image contrast.

\section{Optical properties}

To quantify absorptance, we measure transmittance $(T)$ and reflectance $(R)$ using a UV-vis-NIR spectrophotometer and a FTIR spectrometer. Figure 2(a) shows the difference between the sub-band-gap absorptance $(A=1-T-R)$ of hyperdoped Ge:Au and that of a virgin Ge wafer, $A_{\mathrm{Ge}} \mathrm{Au}-A_{\mathrm{Ge}}$. Figure 2 shows that the Au-doping concentration in the low-dose sample is too small to cause a measurable increase in sub-band-gap absorptance. In contrast, the high-dose sample does show sub-band-gap absorptance.
By modeling the sample as a two-layer thin-film absorbing stack, we can obtain lower and upper bounds for the subband-gap absorption coefficient, $\alpha$, of the high-dose sample from data in Fig. 2. Assuming the hyperdoped $\mathrm{Ge}: \mathrm{Au}$ layer uniformly absorbs sub-band-gap light, we obtain, for sub-band-gap $\alpha$ for hyperdoped $\mathrm{Ge}: \mathrm{Au}$ at a wavelength of $2.4 \mu \mathrm{m}$, an upper bound of $5200 \mathrm{~cm}^{-1}$ (taking the layer thickness to be $50 \mathrm{~nm}$; see Sec. II for details) and a lower bound of $1200 \mathrm{~cm}^{-1}$ (for a 210-nm-thick hyperdoped layer). This range of values is comparable to that of commercially available direct-band-gap semiconductors at the same wavelength $[4,24]$.

We can independently estimate the sub-band-gap $\alpha$ of the high-dose sample from the photoionization cross section of $\mathrm{Ge}: \mathrm{Au}$ and the measured dopant concentration. Sub-band-gap dopant-mediated extrinsic-absorption coefficients scale as $\alpha=\sigma N$, where $\sigma$ is the photoionization cross section and $N$ is the concentration of incorporated dopants [29]. The $A 1, A 2, A 3$, and $D 1 \mathrm{Ge:Au}$ energy levels [19] have photoionization cross sections [29,34] of $4 \times 10^{-17}, 5 \times 10^{-18}, 3 \times 10^{-18}$, and $3 \times 10^{-18} \mathrm{~cm}^{2}$, respectively. Assuming, for simplicity, that (1) the levels do not interact with one another (i.e., ignoring potential band formation) and absorb light independently, (2) only substitutional $\mathrm{Au}$ atoms create mid-band-gap states that absorb sub-band-gap photons (i.e., ignoring possible absorption from interstitials or clusters), (3) all substitutional atoms are optically active, and (4) the average Au-layer substitutional concentration is $2 \times 10^{19} \mathrm{~cm}^{-3}$, the expected extrinsic sub-band-gap $\alpha$ is $1100 \mathrm{~cm}^{-1}$. Alternatively, preserving assumption (1) above, and assuming that (2) both substitutional and interstitial atoms absorb sub-band-gap photons, (3) all these atoms are optically active, and (4) the average-layer total concentration is
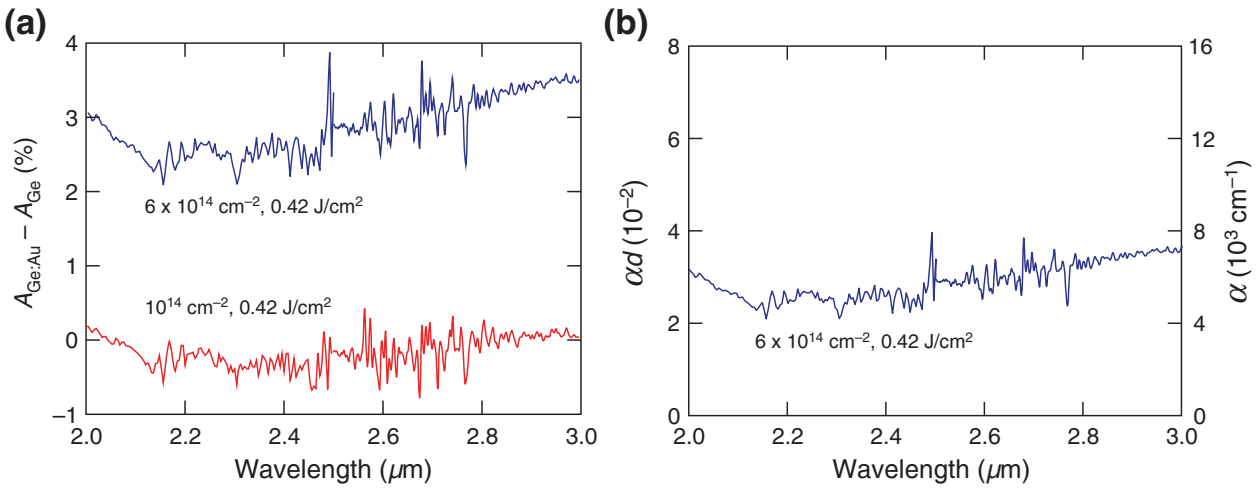

FIG. 2. Sub-band-gap absorptance of hyperdoped Ge. (a) Difference between the sub-band-gap absorptance $A$ (absorptance $=1$-transmittance-reflectance) of hyperdoped Ge samples (processed with a $0.49 \mathrm{~J} / \mathrm{cm}^{2} \mathrm{Nd}$ :YAG laser fluence) and that of the virgin Ge wafer, $A_{\mathrm{Ge}: \mathrm{Au}}-A_{\mathrm{Ge}}$, measured with a UV-NIR spectrophotometer and FTIR. Crossover between spectrophotometer and FTIR spectrometer measurements is at $2.5 \mu \mathrm{m}$. Low-dose $\left(10^{14} \mathrm{~cm}^{-2}\right.$, red line) sample shows no sub-band-gap absorptance, whereas high-dose $\left(6 \times 10^{14} \mathrm{~cm}^{-2}\right.$, blue line) sample shows significant sub-band-gap absorptance. (b) Calculated $\alpha d$ product (left axis) and absorption coefficient (right axis) for the high-dose hyperdoped sample. In estimating the absorption coefficient $\alpha$, the absorber layer thickness, $d$, is approximated as $50 \mathrm{~nm}$. 
$3 \times 10^{19} \mathrm{~cm}^{-3}$, the expected extrinsic sub-band-gap $\alpha$ is approximately $1600 \mathrm{~cm}^{-1}$. Both of these estimates agree well with the lower and upper bounds obtained from measured absorptance data, as discussed above.

We can use the same approach to estimate the expected sub-band-gap $\alpha$ for the low-dose sample, yielding a subband-gap $\alpha$ of approximately $200 \mathrm{~cm}^{-1}$ (using either the substitutional or total concentration). This value is an order of magnitude below that of commercially available direct-band-gap semiconductors at the same wavelength, and therefore, consistent with the lack of sub-band-gap absorption for the low-dose sample observed in Fig. 2.

In Fig. 2(b), we show the nondimensional $\alpha d$ product (left axis) of the high-dose hyperdoped sample, where $d$ is the unknown (effective) thickness of the absorbing hyperdoped layer. The $\alpha d$ product is estimated as described previously [26], using measured transmittance data [shown in Fig. S4(c) within the Supplemental Material [35]], and assuming hyperdoped samples have a reflection coefficient at $2.4 \mu \mathrm{m}$ equivalent to that of a virgin Ge-air interface $(0.366$ at $2.4 \mu \mathrm{m})$. Based on the Au-layer-thickness discussion above, we conclude that an incorporated $\mathrm{Au}$ dose between $3.7 \times 10^{14}$ and $6 \times 10^{14}$ atoms $/ \mathrm{cm}^{-2}$, distributed across a hyperdoped layer of 50-210 nm, produces the measured sub-band-gap $\alpha d$ product. The right axis of Fig. 2(b) shows the absorption coefficient $\alpha$, assuming the absorber layer thickness, $d$, is $50 \mathrm{~nm}$.

\section{Room-temperature sub-band-gap optoelectronic response}

To test whether hyperdoped Ge exhibits a roomtemperature sub-band-gap optoelectronic response, we fabricate prototype photodetectors using the high-dose samples. In the planar device structure, shown in Fig. 3(a), the hyperdoped Ge:Au layer sits atop a raised mesa structure. This structure is isolated from the substrate to reduce leakage currents and to ensure that the applied voltage produces a current through the device's $p^{+}-n$ junction. We choose a planar device structure for its simplicity and potential suitability for SWIR-imaging-array applications. Top and bottom photodetector contacts are ohmic (Fig. S6 within the Supplemental Material [35]). We also fabricate control photodetectors doped with the shallow-level donor Sb. Detailed device configurations for the hyperdoped Ge:Au and Ge:Sb photodetectors are shown in Figs. S6 and S8 within the Supplemental Material [35], respectively. In the Ge:Au photodetector, the hyperdoped Ge:Au layer forms a rectifying junction with the $n$-type substrate, as shown in the dark $I-V$ curve in Fig. 3(b). Because the hyperdoped $\mathrm{Ge}: \mathrm{Au}$ layer forms a rectifying junction only on an $n$-type substrate and not on a $p$-type substrate [compare the dark $I-V$ curves in Fig. 3(b) and Fig. S7(b) within the Supplemental Material [35]], we conclude that holes act as majority carriers in the hyperdoped Ge:Au layer.
We test the photodetectors using the photoconductivity setup shown in Fig. 3(a). We illuminate the photodetectors with chopped light from a continuous-wave laser diode (Brolis semiconductor) at wavelengths of 2.0 and $2.4 \mu \mathrm{m}$. The laser light is mechanically chopped at $23 \mathrm{~Hz}$ and focused on a $20 \mu \mathrm{m}$ spot size in the middle of the mesa-structure surface. Using a lock-in amplifier, we measure the ac current generated between the photodetector's two top bar contacts and the bottom contact. Figure 3(c) shows the difference between the $I-V$ curves obtained under $2.0 \mu \mathrm{m}$ laser illumination and no illumination. This photocurrent difference demonstrates a subband-gap photocurrent. Increasing reverse bias across the junction increases (and then saturates) the sub-band-gap photocurrent due to enhanced collection of charge carriers excited by sub-band-gap light. Under $2.0 \mu \mathrm{m}$ illumination, the Ge:Au photodetector exhibits a responsivity of $2.5 \times 10^{-4} \mathrm{~A} / \mathrm{W}$ and an external quantum efficiency (EQE) of $1.6 \times 10^{-4}$. Under $2.4 \mu \mathrm{m}$ illumination, the device exhibits a responsivity of $2.7 \times 10^{-6} \mathrm{~A} / \mathrm{W}$ and an EQE of $1.4 \times 10^{-6}$.

Figure 3(d) shows the broadband response of the Ge:Au photodetector, which is obtained by substituting the Ge:Au photodetector for the detector in a FTIR spectrometer. The response of each detector is normalized to the intensity spectrum of the FTIR Globar light. Because we do not know the incident power per unit wavelength of the FTIR Globar light illuminating the photodetectors and because the response is obtained from a Fourier transform of the phase-corrected time-domain interferogram, we report the normalized photoconductive response in arb. units. For comparison, we also show the normalized photoconductive response of the Ge:Sb photodetector. Between 2.0 and $3.0 \mu \mathrm{m}$, the response of hyperdoped $\mathrm{Ge}: \mathrm{Au}$ consistently exceeds that of the Ge:Sb photodetector. The dark noise is $2.0 \times 10^{-5}$ for the Ge:Au photodetector and $1.0 \times 10^{-5}$ for the Ge:Sb photodetector. The Ge:Au photodetector exhibits a room-temperature sub-band-gap response up to $3.0 \mu \mathrm{m}$, which is well beyond the detection edge of extended $\mathrm{In}_{1-x} \mathrm{Ga}_{x}$ As (with a band gap of $2.6 \mu \mathrm{m}$ ).

\section{FUTURE WORK}

Figure 3 demonstrates the proof-of-concept roomtemperature sub-band-gap absorption and optoelectronic response in hyperdoped Ge:Au. The low EQE of our device could be increased by optimizing hyperdoping fabrication to enhance sub-band-gap absorptance (i.e., the $\alpha d$ product, given that the current Ge:Au layer we fabricate uses only approximately $3 \%$ of sub-band-gap light) and by maximizing carrier collection through improved device design and fabrication. Four possible paths to enhance device sub-band-gap absorptance are (1) increase the Au-implant dose, (2) increase the Ge:Au layer thickness, (3) optimize the PLM laser fluence, and (4) improve 
(a)

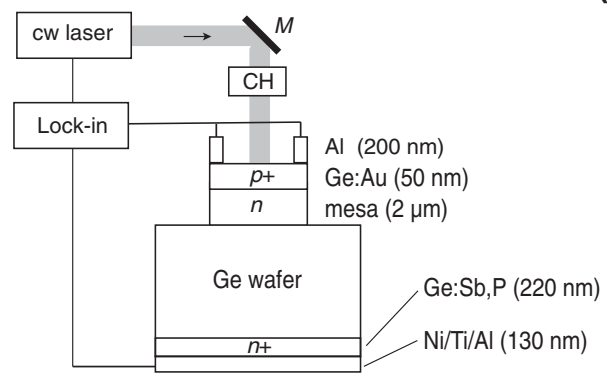

(b)

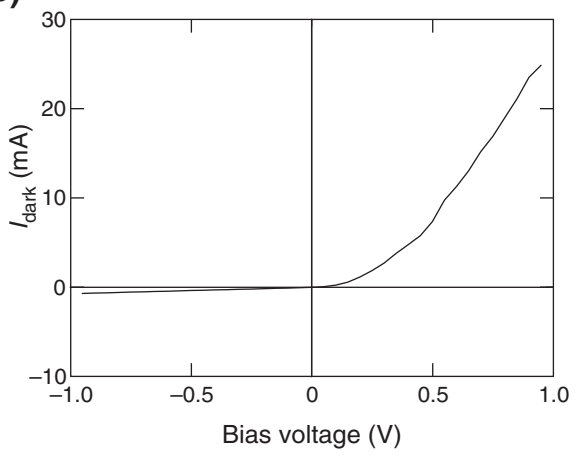

(c)

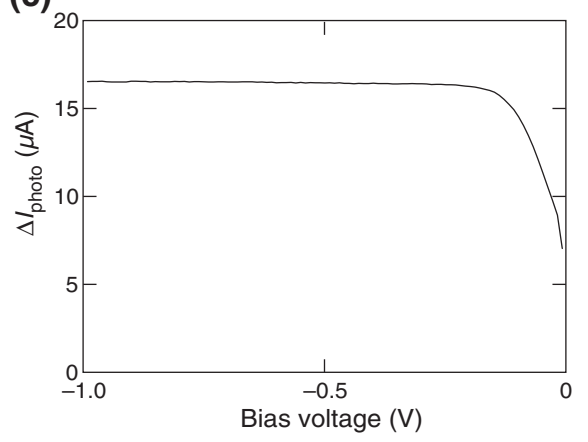

(d)

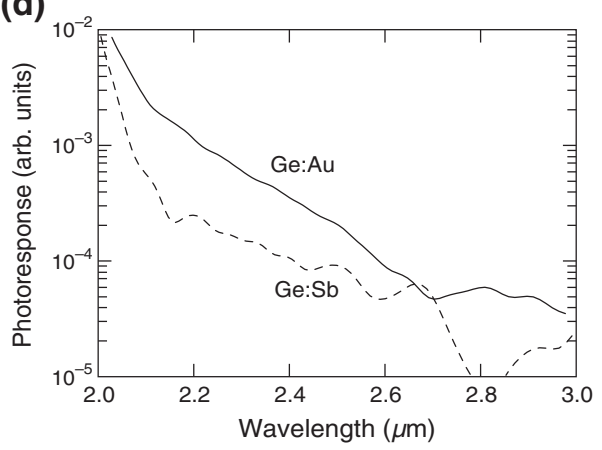

FIG. 3. Room-temperature sub-band-gap optoelectronic response of hyperdoped Ge. (a) Schematic of the cw-laser photoconductivity measurement setup used to measure the optoelectronic response of the high-dose $\left(6 \times 10^{14} \mathrm{~cm}^{-2}\right)$ hyperdoped Ge photodetectors at 2.0 and $2.44 \mu \mathrm{m}$. CH in the diagram stands for chopper. (b) Hyperdoped Ge photodetector's dark $I-V$ curve. (c) Difference between the hyperdoped Ge photodetector's 2.0- $\mu$ m-laser illuminated and dark $I$ - $V$ curves. (d) Measured room-temperature normalized photoresponse of a zero-biased hyperdoped Ge:Au photodetector and Ge:Sb control photodetector also made with implantation and PLM. Response is obtained from substituting each photodetector for the detector in a FTIR spectrometer and illuminating with Globar light. Response of each detector is normalized to the intensity spectrum of the FTIR Globar light.

photon management. Increasing the Au-implant dose would increase the concentration of trapped Au dopants after PLM. This higher Au-dopant concentration, in turn, would increase the dopant-mediated sub-band-gap $\alpha$ in the hyperdoped Ge layer. For a fixed implant dose, increasing the implant energy and laser fluence used to melt the amorphous implanted region increases the thickness, absorptance, and optical path length of the hyperdoped layer. We note that the current hyperdoped-Ge:Au layer thickness, between 50 and $210 \mathrm{~nm}$, can likely be increased. In $\mathrm{Si}$, for example, single-crystal layers with implant doses of $10^{16} \mathrm{~cm}^{-2}$ and thicknesses up to $500 \mathrm{~nm}$ are achieved. Optimizing the PLM laser fluence will increase the substitutional fraction and optical activation of dopants, further increasing sub-band-gap $\alpha$. Preliminary results indicate that laser melting the implanted samples studied here with a lower laser fluence greatly enhances sub-bandgap absorption (Fig. S9 within the Supplemental Material [35]). In addition to optimizing the hyperdoped layer, absorption can be increased through photon management strategies, such as surface structures and antireflection layers. Passivation layers, a smaller device size, and an optimized device architecture could further enhance the EQE. In particular, a future device architecture, where the device depletion layer encompasses as much of the hyperdoped layer as possible, by managing the $p$-type doping or use of MOS structures, for example, may lead to much improved photocollection and higher EQE.

Future development of hyperdoped Ge:Au should also investigate how its properties compare to those of state-ofthe art Ge-Sn alloys. Critical properties to compare should include sub-band-gap absorption (absorption coefficient and wavelength-dependent edge) and properties that currently limit Ge-Sn devices (thermal metastabilty [61,62] and relatively high leakage current $[43,44]$ ) compared with commerical III-V and II-VI semiconductor devices.

\section{CONCLUSION}

We present a scalable method for hyperdoping Ge with $\mathrm{Au}$ that opens the door to low-cost room-temperature Sicompatible SWIR photodetection. Using ion implantation followed by nanosecond PLM, we obtain a single-crystal material with a peak Au concentration of $3 \times 10^{19} \mathrm{~cm}^{-3}$ 
$\left(10^{3}\right.$ times the solubility limit). The high nonequilibrium gold concentrations we obtain give rise to fundamentally different optoelectronic properties than those reported previously at much lower concentrations. The resulting hyperdoped Ge:Au exhibits dopant-mediated sub-bandgap absorption of light up to wavelengths of at least $3 \mu \mathrm{m}$, with an optical absorption coefficient comparable to that of commercial SWIR photodetection materials. In contrast to previous dopant-mediated Ge-based photodetectors, which exhibit sub-band-gap responses only at low temperature, we present a hyperdoped Ge:Au photodetector for sub-band-gap SWIR photodetection that works at room temperature.

\section{ACKNOWLEDGMENTS}

H.H.G. acknowledges support from the Department of Defense (DoD) under Grant No. DGE 0946799 through the National Defense Science and Engineering Graduate Fellowship (NDSEG) Program and the Directed Energy Processing Society Graduate Fellowship. D.P. acknowledges financial support from the MEC within the Programa Nacional de movilidad de recursos humanos del Plan Nacional I + D+i 2008-2011 (Grant No. EX-2010-0662), the program Ramón y Cajal (Grant No. RYC-2014-16936), and from the Spanish ministry of Science, Innovation and Universities (MICINN) under Contract No. TEC201784378-R. This work is part of the project MADRID-PV2 P-2018/EMT-4308 funded by the Regional Government of Madrid with support from FEDER funds. This work is also supported by the US Air Force Office of Scientific Research (Grant No. FA9550-14-1-0150). This work is performed, in part, at the Harvard Center for Nanoscale Systems (CNS), a member of the National Nanotechnology Infrastructure Network (NNIN), which is supported by the National Science Foundation under NSF Grant No. ECS-0335765. All authors thank Brolis semiconductor for use of their SWIR diode lasers for photoconductivity experiments. H.H.G. thanks Austin J. Akey, Meng-Ju Sher, Daniel Recht, Mark Winkler, and Reza Sanatina for feedback during the experimental design process. H.H.G. and D.P. thank Karl K. Berggren and Emily Toomey at MIT for assistance in wire bonding. L.A.S. thanks Austin J. Akey for assistance with XTEM measurements.

H.H.G. and D.P. are co-first authors and contributed equally to this work. H.H.G. and D.P. proposed the initial concept for the study. H.H.G., D.P., T.T.T., J.S.W., M.J.A., and E.M. designed hyperdoping experiments. D.P. performed PLM melting simulations. D.P. and T.T.T. performed SRIM calculations. T.T.T. and J.S.W. performed implantations and RBS measurements. D.P. and H.H.G. performed PLM. D.P., H.H.G., and J.P.M. performed absorptance measurements. T.T.T., E.N., and R.M. performed SIMS measurements. L.A.S. performed XTEM measurements. J.P.M., D.P., and H.H.G. decided on photodetector device design. D.P and H.H.G. fabricated photodetectors. S.K. and D.P. performed photoconductivity experiments. H.H.G. prepared the manuscript. H.H.G., D.P., and T.T.T. prepared manuscript figures. E.M. and M.J.A. supervised the work and development of the manuscript.

[1] Mark P. Hansen and Douglas S Malchow, in Overview of SWIR (2008).

[2] R. G. Driggers, V. Hodgkin, and R. Vollmerhausen, in What Good Is SWIR? Passive Day Comparison of VIS, NIR, and $S W I R$, edited by G. C. Holst, K. A. Krapels (2013), pp. 87060L.

[3] M. Lothode, V. Carrere, and R. Marion, in Identifying Industrial Processes Through VNIR-SWIR Reflectance Spectroscopy of Their Waste Materials (IEEE, 2014), pp. 3288-3291.

[4] A. Rogalski, Infrared detectors: Status and trends, Prog. Quantum Electron. 27, 59 (2003).

[5] R. Soref, Enabling $2 \mu \mathrm{m}$ communications, Nat. Photonics 9, 358 (2015).

[6] T. E. Kazior, J. R. LaRoche, D. Lubyshev, J. M. Fastenau, W. K. Liu, M. Urteaga, W. Ha, J. Bergman, M. J. Choe, M. T. Bulsara, E. A. Fitzgerald, D. Smith, D. Clark, R. Thompson, C. Drazek, N. Daval, L. Benaissa, and E. Augendre, in Progress and Challenges in the Direct Monolithic Integration of III-V Devices and Si CMOS on Silicon Substrates (IEEE, 2009), pp. 100-104.

[7] T. E. Kazior, Beyond CMOS: Heterogeneous integration of III-V devices, RF MEMS and other dissimilar materials/devices with Si CMOS to create intelligent microsystems, Philos. Transact. A Math. Phys. Eng. Sci. 372 (2014).

[8] J. Guo, Y. Liu, Y. Ma, E. Zhu, S. Lee, Z. Lu, Z. Zhao, C. Xu, S.-J. Lee, H. Wu, K. Kovnir, Y. Huang, and X. Duan, Few-Layer GeAs field-effect transistors and infrared photodetectors, Adv. Mater. 30, 1705934 (2018).

[9] X. Tian, H. Luo, R. Wei, C. Zhu, Q. Guo, D. Yang, F. Wang, J. Li, and J. Qiu, An ultrabroadband Mid-infrared pulsed optical switch employing solution-processed bismuth oxyselenide, Adv. Mater. 30, 1801021 (2018).

[10] X. Wang, A. Yakovliev, T. Y. Ohulchanskyy, L. Wu, S. Zeng, X. Han, J. Qu, and G. Chen, Efficient erbiumsensitized core/shell nanocrystals for short wave infrared bioimaging, Adv. Opt. Mater. 6, 1800690 (2018).

[11] F. H. L. Koppens, T. Mueller, Ph. Avouris, A. C. Ferrari, M. S. Vitiello, and M. Polini, Photodetectors based on graphene, other Two-dimensional materials and hybrid systems, Nat. Nanotechnol. 9, 780 (2014).

[12] T. Reiss, K. Hjelt, and A. C. Ferrari, Graphene Is on track to deliver on Its promises, Nat. Nanotechnol. 14, 907 (2019).

[13] W. Dong, Y. Qiu, X. Zhou, A. Banas, K. Banas, M. B. H. Breese, T. Cao, and R. E. Simpson, Tunable Mid-infrared phase-change metasurface, Adv. Opt. Mater. 6, 1701346 (2018).

[14] K.-T. Lin, H.-L. Chen, Y.-S. Lai, and C.-C. Yu, SiliconBased broadband antenna for high responsivity and polarization-insensitive photodetection at telecommunication wavelengths, Nat. Commun. 5, 3288 (2014). 
[15] R. Saran and R. J. Curry, Lead sulphide nanocrystal photodetector technologies, Nat. Photonics 10, 81 (2016).

[16] S. Park, K. Fukuda, M. Wang, C. Lee, T. Yokota, H. Jin, H. Jinno, H. Kimura, P. Zalar, N. Matsuhisa, S. Umezu, G. C. Bazan, and T. Someya, Ultraflexible near-infrared organic photodetectors for conformal photoplethysmogram sensors, Adv. Mater. 30, 1802359 (2018).

[17] Q. Shen, Z. Luo, S. Ma, P. Tao, C. Song, J. Wu, W. Shang, and T. Deng, Bioinspired infrared sensing materials and systems, Adv. Mater. 30, 1707632 (2018).

[18] M. Casalino, G. Coppola, R. M. D. L. Rue, and D. F. Logan, State-of-the-Art All-silicon Sub-bandgap photodetectors at telecom and datacom wavelengths, Laser Photonics Rev. 10, 895 (2016).

[19] T. Pham, W. Du, H. Tran, J. Margetis, J. Tolle, G. Sun, R. A. Soref, H. A. Naseem, B. Li, and S.-Q. Yu, Systematic study of Si-based GeSn photodiodes with $2.6 \mu \mathrm{m}$ detector cutoff for short-wave infrared detection, Opt. Express 24, 4519 (2016).

[20] V. Reboud, A. Gassenq, J. M. Hartmann, J. Widiez, L. Virot, J. Aubin, K. Guilloy, S. Tardif, J. M. Fédéli, N. Pauc, A. Chelnokov, and V. Calvo, Germanium based photonic components toward a full silicon/germanium photonic platform, Prog. Cryst. Growth Charact. Mater. 63, 1 (2017).

[21] K. P. Homewood and M. A. Lourenço, Optoelectronics: The rise of the GeSn laser, Nat. Photonics 9, 78 (2015).

[22] J. M. Warrender, Laser hyperdoping silicon for enhanced infrared optoelectronic properties, Appl. Phys. Rev. 3, 031104 (2016).

[23] C. Claeys and E. Simoen, Germanium-Based Technologies: From Materials to Devices, 1 edition (Elsevier Science, Amsterdam; Boston, 2007).

[24] A. Rogalski, Recent progress in infrared detector technologies, Infrared Phys. Technol. 54, 136 (2011).

[25] J. J. Ackert, D. J. Thomson, L. Shen, A. C. Peacock, P. E. Jessop, G. T. Reed, G. Z. Mashanovich, and A. P. Knights, High-Speed detection at Two micrometres with monolithic silicon photodiodes, Nat. Photonics 9, 393 (2015).

[26] J. P. Mailoa, A. J. Akey, C. B. Simmons, D. Hutchinson, J. Mathews, J. T. Sullivan, D. Recht, M. T. Winkler, J. S. Williams, J. M. Warrender, P. D. Persans, M. J. Aziz, and T. Buonassisi, Room-Temperature Sub-band Gap optoelectronic response of hyperdoped silicon, Nat. Commun. 5 (2014).

[27] W. Yang, N. Ferdous, P. J. Simpson, J. M. Gaudet, Q. Hudspeth, P. K. Chow, J. M. Warrender, A. J. Akey, M. J. Aziz, E. Ertekin, and J. S. Williams, Evidence for vacancy trapping in Au-hyperdoped Si following pulsed laser melting, APL Mater. 7, 101124 (2019).

[28] W. Yang, Q. Hudspeth, P. K. Chow, J. M. Warrender, N. Ferdous, E. Ertekin, G. Malladi, A. J. Akey, M. J. Aziz, and J. S. Williams, Atomistic Mechanisms for the Thermal Relaxation of Au-Hyperdoped Si, Phys. Rev. Appl. 12, 024015 (2019).

[29] E. H. Putley and Far Infra-Red Photoconductivity. Phys. Status Solidi B 6, 571 (1964).

[30] N. Sclar, Properties of doped silicon and germanium infrared detectors, Prog. Quantum Electron. 9, 149 (1984).

[31] J. Zhu, H. Zhu, H. Xu, Z. Weng, and H. Wu, GeBased Mid-infrared blocked-impurity-band photodetectors, Infrared Phys. Technol. 92, 13 (2018).
[32] J. W. Beeman, S. Goyal, L. A. Reichertz, and E. E. Haller, Ion-Implanted Ge:B Far-infrared blockedimpurity-band detectors, Infrared Phys. Technol. 51, 60 (2007).

[33] J. Bandaru, J. W. Beeman, E. E. Haller, S. Samperi, and N. M. Haegel, Influence of the Sb dopant distribution on Far infrared photoconductivity in Ge:Sb blocked impurity band detectors, Infrared Phys. Technol. 43, 353 (2002).

[34] S. M. Sze and K. K. Ng, Physics of Semiconductor Devices, 3rd ed (Wiley-Interscience, Hoboken, N.J, 2007).

[35] See the Supplemental Material at http://link.aps.org/sup plemental/10.1103/PhysRevApplied.14.064051 for Ge:Au defect levels, laser-melting schematic and simulations, Raman spectra, additional absorptance data, additional $I-V$ curves, and normalized SIMS profiles.

[36] C. B. Simmons, A. J. Akey, J. P. Mailoa, D. Recht, M. J. Aziz, and T. Buonassisi, Enhancing the infrared photoresponse of silicon by controlling the Fermi level location within an impurity band, Adv. Funct. Mater. 24, 2852 (2014).

[37] P. M. Smith and M. J. Aziz, Solute trapping in aluminum alloys, Acta Metall. Mater. 42, 3515 (1994).

[38] L. Johnson and H. Levinstein, Infrared properties of gold in germanium, Phys. Rev. 117, 1191 (1960).

[39] R. A. Soref and L. Friedman, Direct-Gap Ge/GeSn/Si and $\mathrm{GeSn} / \mathrm{Ge} / \mathrm{Si}$ heterostructures, Superlattices Microstruct. 14, 189 (1993).

[40] R. Geiger, T. Zabel, and H. Sigg, Group IV direct band Gap photonics: Methods, challenges, and opportunities, Front. Mater. 2 (2015).

[41] J. Mathews, R. Roucka, J. Xie, S.-Q. Yu, J. Menéndez, and J. Kouvetakis, Extended performance GeSn/Si(100) p-i-n photodetectors for full spectral range telecommunication applications, Appl. Phys. Lett. 95, 133506 (2009).

[42] H. Tran, T. Pham, W. Du, Y. Zhang, P. C. Grant, J. M. Grant, G. Sun, R. A. Soref, J. Margetis, J. Tolle, B. Li, M. Mortazavi, and S.-Q. Yu, High performance $\mathrm{Ge}_{0.89} \mathrm{Sn}_{0.11}$ photodiodes for Low-cost shortwave infrared imaging, J. Appl. Phys. 124, 013101 (2018).

[43] H. Tran, T. Pham, J. Margetis, Y. Zhou, W. Dou, P. C. Grant, J. M. Grant, S. Al-Kabi, G. Sun, R. A. Soref, J. Tolle, Y.H. Zhang, W. Du, B. Li, M. Mortazavi, and S.-Q. Yu, SiBased GeSn photodetectors toward Mid-infrared imaging applications, ACS Photonics 6, 2807 (2019).

[44] C. L. Senaratne, P. M. Wallace, J. D. Gallagher, P. E. Sims, J. Kouvetakis, and J. Menéndez, Direct Gap $G e_{1-y} S n_{y}$ alloys: Fabrication and design of Mid-IR photodiodes, J. Appl. Phys. 120, 025701 (2016).

[45] S. Wirths, R. Geiger, N. von den Driesch, G. Mussler, T. Stoica, S. Mantl, Z. Ikonic, M. Luysberg, S. Chiussi, J. M. Hartmann, H. Sigg, J. Faist, D. Buca, and D. Grützmacher, Lasing in direct-bandgap GeSn alloy grown on $\mathrm{Si}$, Nat. Photonics 9, 88 (2015).

[46] J. Michel, J. Liu, L. C. Kimerling, and High-Performance Ge-on-Si Photodetectors. Nat. Photonics 4, 527 (2010).

[47] D. Pastor, H. H. Gandhi, C. P. Monmeyran, A. J. Akey, R. Milazzo, Y. Cai, E. Napolitani, R. M. Gwilliam, I. F. Crowe, J. Michel, L. C. Kimerling, A. Agarwal, E. Mazur, and M. J. Aziz, High level active N+ doping of strained germanium through Co-implantation and nanosecond pulsed laser melting, J. Appl. Phys 123, 165101 (2018). 
[48] G. Impellizzeri, S. Mirabella, and M. G. Grimaldi, Ion implantation damage and crystalline-amorphous transition in Ge, Appl. Phys. A 103, 323 (2011).

[49] R. G. Elliman and J. S. Williams, Advances in Ion beam modification of semiconductors, Curr. Opin. Solid State Mater. Sci 19, 49 (2015).

[50] H. S. Alkhaldi, T. T. Tran, F. Kremer, and J. S. Williams, The influence of capping layers on pore formation in Ge during Ion implantation, J. Appl. Phys. 120, 215706 (2016).

[51] T. T. Tran, H. S. Alkhaldi, H. H. Gandhi, D. Pastor, L. Q. Huston, J. Wong-Leung, M. J. Aziz, and J. S. Williams, Suppression of Ion-implantation induced porosity in germanium by a silicon dioxide capping layer, Appl. Phys. Lett. 109, 082106 (2016).

[52] International atomic energy agency, Electronic Stopping Power of Matter for Ions, https://www-nds.iaea.org/stop ping/.

[53] D. H. Auston, C. M. Surko, T. N. C. Venkatesan, R. E. Slusher, and J. A. Golovchenko, Time-resolved reflectivity of Ion-implanted silicon during laser annealing, Appl. Phys. Lett. 33, 437 (1978).

[54] J. S. Williams and R. G. Elliman, Ion Beams for Materials Analysis (Academic Press, San Diego, USA, 1989).

[55] B. Yang, J.-J. Lin, S. Gupta, A. Roy, S. Liang, W. P. Maszara, Y. Nishi, and K. Saraswat, in 2012 International Silicon-Germanium Technology and Device Meeting (ISTDM) (2012), pp. 1-2.
[56] "LIMP, for Laser Induced Melting Predictions, Is a Numerical Solution to the 1-D Heat Equation for PLM. It Was Originally Written by Michael O. Thompson and Modified by Jeffrey A. West, Patrick Michael Smith and David E. Hoglund. A Partial Description Can Be Found in D.E. Hoglund, M.O. Thompson and M.J. Aziz, 'Experimental Test of Morphological Stability Theory for a Planar Interface during Rapid Solidification', Physical Review B 58, 189-199 (1998)” (n.d.).

[57] M. J. Aziz, J. Y. Tsao, M. O. Thompson, P. S. Peercy, and C. W. White, Solute trapping: Comparison of Theory with Experiment, Phys. Rev. Lett. 56, 2489 (1986).

[58] M. Mayer, SIMNRA, a Simulation Program for the Analysis of NRA, RBS and ERDA, in AIP Conference Proceedings, Vol. 475 (1999), p. 541.

[59] S. Rubanov and P. R. Munroe, The application of FIB milling for specimen preparation from crystalline germanium, Micron 35, 549 (2004).

[60] N. Yao, Focused Ion Beam Systems: Basics and Applications (Cambridge University Press, New York, 2007).

[61] N. Bhargava, J. P. Gupta, N. Faleev, L. Wielunski, and J. Kolodzey, Thermal stability of annealed germaniumTin alloys grown by molecular beam epitaxy, J. Electron. Mater. 46, 1620 (2017).

[62] D. Schwarz, H. S. Funk, M. Oehme, and J. Schulze, Alloy stability of $G e_{1-x} S n_{x}$ with $\mathrm{Sn}$ concentrations up to $17 \%$ utilizing Low-temperature molecular beam epitaxy, J. Electron. Mater. 49, 5154 (2020). 